\title{
EXTENSION OF A DISTRIBUTIVE LATTICE TO A BOOLEAN RING*
}

\author{
H. M. MacNEILLE
}

The problem of imbedding an abstract distributive lattice in a Boolean algebra by an algebraic extension was suggested to the writer by M. H. Stone in 1933. Hausdorff $\dagger$ had already given a solution of this problem for the case where the given distributive lattice was a ring of point sets. A solution for the abstract case was presented by the writer to the Harvard Mathematical Colloquium (1934), included in his doctoral dissertation, and published. $\ddagger$

In the meantime, Stone $\S$ had discovered that a Boolean algebra is a special type of algebraic ring. This revealed many properties of Boolean algebras to be instances of known ring properties and led the writer to believe that the imbedding of a distributive lattice in a Boolean algebra might be subsumed under some well established algebraic procedure. This was found to be the case. $\|$ For, if a hypercomplex system is constructed upon the given distributive lattice as a basis with the integers modulo 2 as coefficient field, the resulting ring, reduced by an ideal, is the required extension. This construction is presented in this paper. Aside from the unification it achieves, it is shorter and more elegant than previous solutions.

An algebraic ring $\llbracket$ in which every element is idempotent with re-

* Presented to the Society, September 3, 1936, under the title Extension of a multiplicative system to a Boolean ring.

$\dagger$ F. Hausdorff, Mengenlehre, 2d edition, Leipzig, de Gruyter, 1927, p. 79. Hausdorff's solution uses the assumption that the elements are point sets in defining equality. Garrett Birkhoff has proved (Proceedings of the Cambridge Philosophical Society, vol. 29 (1933), pp. 441-464, Theorem 25.2) that every distributive lattice can be represented by a ring of point sets. Together, the work of Hausdorff and Birkhoff establishes the desired conclusion. The object of Stone's suggestion was to avoid the use of transfinite induction upon which Birkhoff's proof depends.

$\ddagger$ H. M. MacNeille, Extensions of Partially Ordered Sets, doctoral dissertation, Harvard University, 1935; Proceedings of the National Academy of Sciences, vol. 22 (1936), pp. 45-50; Transactions of this Society, vol. 42 (1937), pp. 416-460.

$\S$ M. H. Stone, Proceedings of the National Academy of Sciences, vol. 21 (1935), pp. 103-105.

\| H. M. MacNeille, this Bulletin, abstract 42-7-316.

I For the definition and elementary properties of algebraic rings and hypercomplex systems, see B. L. van der Waerden, Moderne Algebra, Berlin, Springer, 1931, vol. 1, chap. 3, and vol. 2, chap. 16. For rings and fields of point sets, see Hausdorff, loc. cit., $\$ 17$. For Boolean rings, see M. H. Stone, Transactions of this Society, vol. 40 (1936), pp. 37-111, chap. 1. For distributive lattices, see M. H. Stone, Czecho- 
spect to multiplication $(a a=a)$ is called a Boolean ring. Such a ring is necessarily commutative $(a b=b a)$, and addition is modulo 2 $(a+a=0)$. A Boolean ring may, but need not, contain a unit for multiplication. In any Boolean ring, a third operation $a \vee b$ is defined by the equation

$$
a \vee b=a+b+a b .
$$

When the Boolean ring is a field of point sets, $a v b$ is the union of $a$ and $b$. Furthermore, in any Boolean ring, $a \vee b$ enjoys the algebraic properties of point set union and will be referred to as the union of $a$ and $b$ even when $a$ and $b$ are not point sets.

Regarding union and multiplication as the basic operations, every Boolean ring is a distributive lattice. It is natural to ask whether the definition (1) of union can be inverted so as to define addition in terms of union and multiplication. However, one finds this not to be the case. Not every distributive lattice is a Boolean ring.* Closure with respect to addition and multiplication implies the existence of relative complements, while closure with respect to union and multiplication does not. That is, if $a$ and $b$ are elements of a Boolean ring, an element $x$ (namely $b+a b$ ) exists such that $a \vee x=a \vee b$ and $a x=0$, while the distributive lattice operations of union and multiplication do not require such an element. Thus the problem of imbedding a distributive lattice in a Boolean ring is not vacuous.

Let a distributive lattice $K$ with elements $a, b, c, \cdots$, and operations of union and multiplication be given. Consider the hypercomplex system $H$ generated by $K$ as basis and with the integers modulo 2 as coefficient field. Equal elements in $K$ are regarded as identical. Multiplication of basis elements is taken as the given lattice multiplication. In many cases, the rank of $H$ will be infinite, but elements of $H$ are allowed only a finite number of coefficients different from zero. Elements of $H$ can be represented as sums $\left(\sum_{i=1}^{n} a_{i}=a_{1}+a_{2}+a_{3}+\cdots+a_{n}\right)$ of a finite number of elements of $K$, where inclusion in the summation indicates that the coefficient is 1 and omission from the summation indicates that the coefficient is 0 . Furthermore, since equal elements in $K$ are regarded as identical, no two summands are equal. Two elements of $H$ are equal if they have

slovakian Journal for the Advancement of Mathematics and Physics, vol. 67 (1937), no. 1, pp. 1-25, \$1, and Birkhoff, C lattices, loc. cit. The present paper follows the notation of Stone.

* For example, if union is defined as least upper bound and multiplication as greatest lower bound, the real number system is a distributive lattice but not a Boolean ring. 
the same summands. Each summand is called a component of the element. $H$ contains the element all of whose coefficients are 0 . This is the zero element in $H$ and will be denoted by 0 . The system $H$ is a ring, and every element of $H$ is idempotent with respect to multiplication; therefore we have the following theorem:

Theorem. $H$ is a Boolean ring.

The lattice $K$ is imbedded in $H$, as each element of $K$ is a basis element of $H$. Equality and multiplication in $K$ correspond to equality and multiplication in $H$. If $K$ contains a zero element, it is the element of $H$ with itself as sole component. The zero of $H$ has no components. These two elements form an ideal in $H$. Reducing $H$ by this ideal identifies the zero of $K$ with the zero of $H$ and has no other effect. From this point on, we assume that, if $K$ has a zero, it has been identified with the zero of $H$ and never occurs as a component of an element of $H$.

Equation (1) defines union in $H$, but this definition does not correspond with union as defined in $K$. This is evident from the fact that the union in $H$ of two elements in $K$ has three components while the union in $K$ of these elements has but one component in $H$. Let $M$ be the subring of $H$ generated by all elements of the form $(a \vee b)+a+b+a b$, where $a \vee b$ is the union in $K$ of $a$ and $b$. The subring $M$ is an ideal in $H$. Let $L$ be the quotient ring $H / M$. The ring $L$ is a Boolean ring in which $K$ is imbedded and (1) holds when the indicated union is performed in $K$. That is, the operation of union as given in $K$ corresponds with union as defined in terms of addition and multiplication in $L$. The reduction of $H$ by $M$ does not introduce new equalities in $K$. For, if $a \neq b$ in $K$, then $a+b \neq 0$ in $H$, and $a+b$ does not belong to $M$. Therefore, $a+b \neq 0$ in $L$ and $a \neq b$ in $L$.

It remains to show that $L$ is the smallest Boolean ring in which $K$ can be imbedded. That is, we must show that any reduction of $L$ imposes new equalities on $K$. As a first step, a standard form is found for the elements of $L$. An element $\sum_{i=1}^{n} a_{i}$ of $L$ is said to be in standard form if $a_{i} a_{j}=a_{j}$ whenever $i \leqq j$.

Lemma. Every element of $L$ can be expressed in standard form.

The proof is by induction on $n$ and successive applications of the identity $a+b=(a \vee b)+a b$. Since $(a \vee b) a b=a b$, this identity itself establishes the lemma for $n=2$. Assuming the lemma established for $n=k$, consider $\sum_{l-1}^{k+1} a_{i}$ where the last $k$ summands are assumed in standard form. Applying the identity $k$ times to pairs of adjacent summands starting with the first and second, and increasing indices 
by one each time, one obtains

$$
\sum_{i=1}^{k+1} a_{i}=\sum_{i=1}^{k}\left(a_{1} a_{i} \vee a_{i+1}\right)+a_{1} a_{k+1}
$$

where the right-hand member of the equation is in standard form.

LEMMA. If $\sum_{i=1}^{n} a_{i},(n \neq 0)$, is in standard form, the assumption that $\sum_{i=1}^{n} a_{i}=0$ imposes new equalities on $K$.

If $n$ is odd, multiplying through by $a_{n}$, one obtains $a_{n} \sum_{i=1}^{n} a_{i}=a_{n}=0$ which implies $a_{n}=0$ in $K$, contrary to hypothesis; and if $n$ is even, multiplying through by $a_{n-1}$, one obtains $a_{n-1} \sum_{i=1}^{n} a_{i}=a_{n-1}+a_{n}=0$ which implies $a_{n-1}=a_{n}$ in $K$, contrary to hypothesis.

The following theorems are immediate consequences of this lemma.

Theorem. An element of $L$ in standard form is equal to zero only if it is identically zero.

Theorem. $L$ is the smallest Boolean ring in which $K$ can be imbedded.

Kenyon College 